\title{
Comment on Microsurgical therapy of pituitary adenomas
}

\author{
Michael Buchfelder ${ }^{1}$
}

Received: 2 November 2017 / Accepted: 2 February 2018 / Published online: 15 May 2018

(c) Springer Science+Business Media, LLC, part of Springer Nature 2018

In this article [1], Mortini et al. review their extensive experience with the surgical treatment of pituitary adenomas. Their results, in terms of tumor resection and normalization rates of hormonal oversecretion are outstanding, while the complication rate is minimal. They extend a similar, previous report [2] to larger patient numbers and longer follow-up periods. The data presented convincingly show, which excellent results can be achieved with a microsurgical technique by an experienced surgeon in a specialized center with a high case load. Moreover, even the rare entities of pituitary adenomas, such as thyrotropinomas, are represented in impressive numbers [1]. The data furthermore show that adaption of the endoscopic method is not necessarily required, that in contrast, impressive results can be obtained by using microsurgical excellency and that microsurgery is not at all outdated in the therapy of pituitary tumors. Virtually all endoscopic series lack such high patient numbers and long-term outcome data. The authors can be congratulated not only to the remarkable outcome but also to this concise and well written publication that might serve as a reference for to date and even in the near future.

\section{References}

1. P. Mortini, et al., Microsurgical therapy of pituitary adenomas. Endocrine (2017). https://doi.org/10.1007/s12020-017-1458-3

2. P. Mortini, M. Losa, R. Barzagi, N. Boari, M. Giovanelli, Results of transsphenoidal surgery in a large series of patiens with pituitary adenoma. Neurosurgery 56, 1222-1233 (2005)
Michael Buchfelder

michael.buchfelder@uk-erlangen.de

1 Neurochirurgische Klinik, Universitätsklinikum Erlangen, Erlangen, Germany 